\title{
Morphological identification of the patterns of prostatic intraepithelial neoplasia and their importance
}

\author{
Rodolfo Montironi, Roberta Mazzucchelli, Ferran Algaba, Antonio Lopez-Beltran
}

\begin{abstract}
High grade prostatic intraepithelial neoplasia (PIN) is the most likely precursor of prostatic carcinoma. PIN has a high predictive value as a marker for carcinoma, and its identification in biopsy specimens warrants repeat biopsy for concurrent or subsequent carcinoma. The only methods of detection are biopsy and transurethral resection; PIN does not greatly raise the concentration of serum prostate specific antigen (PSA) or its derivatives, does not induce a palpable mass, and cannot be detected by ultrasound. Androgen deprivation decreases the prevalence and extent of PIN, suggesting that this form of treatment might play a role in chemoprevention. Radiotherapy is also associated with a decreased incidence of PIN.

(F Clin Pathol 2000;53:655-665)
\end{abstract}

Keywords: prostate; prostatic intraepithelial neoplasia; intraductal dysplasia; intraductal carcinoma; atypical adenomatous hyperplasia; prostatic adenocarcinoma; chemoprevention

Prostatic intraepithelial neoplasia (PIN; synonyms: intraductal dysplasia; severe dysplasia; large acinar atypical hyperplasia; duct-acinar dysplasia $)^{1}$ is defined by an intraluminal proliferation of the secretory cells of the prostate duct-acinar system. PIN is characterised by a spectrum of atypical cytological features ranging from minimal changes to those that are indistinguishable from carcinoma. A basal cell layer consistently envelopes this intraduct/ acinar proliferation.

Initial references to such lesions were probably made by Orteil ${ }^{2}$ and Andrews. ${ }^{3}$ In $1965 \mathrm{McNeal}^{4}$ emphasised the possible premalignant nature of this proliferative change and, with Bostwick in $1986,{ }^{5}$ described diagnostic criteria for its recognition (the term "intraductal dysplasia" was used by these two authors) and introduced a three grade classification system. Bostwick and Brawer ${ }^{6}$ proposed the term PIN, which was promulgated in 1989 at a workshop on prostate preneoplastic lesions sponsored by the American Cancer Society. The conference recommended modification of the PIN classification as either low grade or high grade PIN to replace PIN1 and PIN2 plus PIN3, respectively.

The concept of PIN has changed dramatically in recent years. An enormous amount of knowledge has been gathered about a variety of intraductal and intra-acinar proliferations, in- dicating that the term PIN might represent a heterogeneous group of lesions. The pathologist has a fundamental role in the pattern identification of this variety of prostate intraductal and intra-acinar lesions.

In our paper, we review the features of high grade PIN as originally described with its various micro-architectural patterns, the morphological subgroups subsequently recognised, changes in high grade PIN after treatment, and the possibility of a preinvasive lesion with molecular but not morphological abnormalities. Finally, we consider the question of whether high grade PIN can be distinguished from intraduct/acinar spread of an invasive prostatic carcinoma.

\section{Identification of high grade PIN \\ HISTOLOGICAL CRITERIA}

The classification of PIN into low grade and high grade is based mainly on the cytological characteristics of the cells. The nuclei of cells composing low grade PIN are enlarged, vary in size, have a normal or slightly increased chromatin content, and possess small or inconspicuous nucleoli (figs 1 and 2). High grade PIN is characterised by cells with large nuclei of relatively uniform size, an increased chromatin content, which might be irregularly distributed, and prominent nucleoli that are similar to those of carcinoma cells (fig 3). The basal cell layer is intact or rarely interrupted in low grade PIN, but may have frequent disruptions in high grade lesions. Although the cytological features of low grade and high grade PIN are fairly constant, the architecture shows a spectrum, varying from a flattened epithelium to a florid cribriform proliferation. Four basic patterns that often coexist have been described by Bostwick and colleagues ${ }^{7}$ : flat, tufting, micropapillary, and cribriform. Familiarity with this diverse architectural spectrum may facilitate the histological recognition of PIN, even though these various architectural patterns have no apparent clinicopathological relevance.

Neuroendocrine differentiation occurs in PIN, where it is intermediate in degree between normal prostate (which has the most cells with neuroendocrine differentiation) and carcinoma. ${ }^{8} 9$ Paneth cell like change of the prostatic epithelium (neuroendocrine cells with large eosinophilic granules) is considered to be a distinct form of neuroendocrine differentiation characterised by isolated cells or small groups of cells with prominent eosinophilic cytoplasmic granules. 


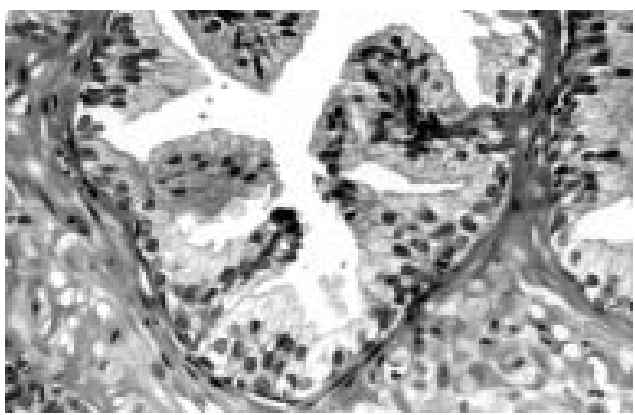

Figure 1 Normal prostate. The duct is lined by a two cell layer-for example, the basal cell and the secretory or lumenal cell layers.

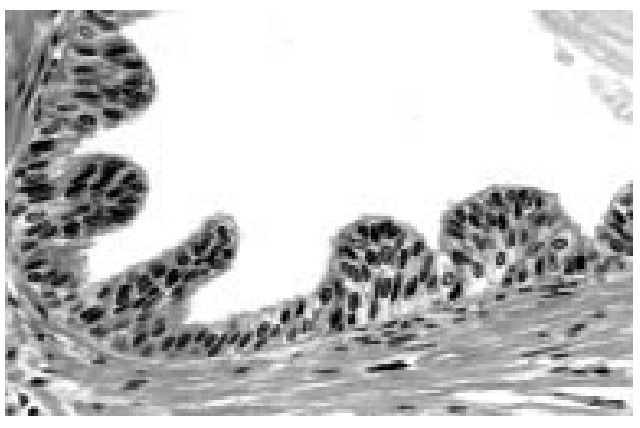

Figure 2 Low grade prostatic intraepithelial neoplasia. The nuclei of the secretory cells are enlarged, vary in size, have a normal or slightly increased chromatin content, and possess small or inconspicuous nucleoli. The basal cell layer is almost intact.

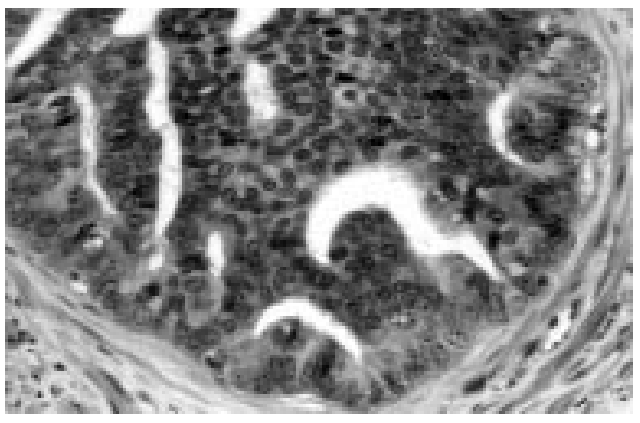

Figure 3 High grade prostatic intraepithelial neoplasia with cribriform pattern. The perimeter cells show features of clearly dysplastic cells, whereas, going from the periphery towards the centre, the nuclei become smaller and the nucleoli become less apparent ("maturation phenomenon"). The basal cell layer is disrupted.

The neuroendocrine products of these cells have local growth promoting and possibly anti-apoptotic activity on the epithelial and endothelial cells. These findings raise the possibility that neuroendocrine differentiation could be involved in the transition from PIN to invasive carcinoma. ${ }^{10-12}$

\section{REPRODUCIBILITY}

Epstein and colleagues ${ }^{13}$ and Allam and colleagues ${ }^{14}$ have looked specifically at the issue of interobserver and intraobserver variability in the identification of PIN. In the study published by the former group none of the participating pathologists diagnosed PIN1 (or low grade PIN) on needle biopsy or transurethral resection of the prostate (TURP). Furthermore, even if a lesion possibly qualifying as low grade PIN was found on needle biopsy, none of the pathologists looked at additional levels in the block or processed the remaining material from the TURP specimen. The rational for not commenting on PIN1 was twofold. First, the distinction of PIN1 from benign prostate glands is often subjective. There was no case for which there was a uniform diagnosis of PIN1; in all cases at least some pathologists considered the biopsies normal. This result is similar to that reported by Tsuchiya et $a l,{ }^{15}$ where the lowest consensus was for lesions considered normal and PIN1. The other reason for not commenting on PIN1 was that, when PIN1 is diagnosed on needle biopsy and repeat biopsies are performed, the patients are at no greater risk of having cancer than when their initial biopsy is reported as benign prostate tissue.

The crucial distinction is between low and high grade PIN. Although some authors combine PIN1 and PIN2 as low grade PIN, it is the consensus of most experts in the literature that PIN2 and PIN3 should be considered to be high grade PIN. In the study performed by Epstein et al, ${ }^{13}$ reproducibility was better when PIN2 and PIN3 were combined compared with combining PIN1 and PIN2. There are several reasons why PIN2 and PIN3 should be combined as high grade PIN. First, there is much interobserver variability in the distinction between PIN2 and PIN $3 .{ }^{13}$ In addition, regardless of whether PIN2 or PIN3 is diagnosed on needle biopsy, both are associated with the same risk of the patient having prostatic carcinoma on subsequent biopsy. ${ }^{16}$

Cases in which there was discrepancy between low grade and high grade fell into at least two groups. ${ }^{13}$ One included cases with prominent cytological atypia but a lack of prominent nucleoli; in the other group the lesion was extensive or stood out at low magnification but prominent nucleoli were not evident. An issue that may lead to discrepant diagnoses between low and high grade PIN is the definition of "prominent" used when describing nucleolar enlargement and visibility.

Another situation in which pathologists disagree is the issue of whether the case is high grade PIN or cancer. ${ }^{13}$ This is particularly true in the presence of cribriform glands. Cribriform glands raise the differential diagnosis with both cribriform acinar and cribriform ductal prostatic carcinoma. The former may be composed of back to back glands or large irregular glands inconsistent with high grade PIN. Ductal carcinoma may also have extensive necrosis and/or papillary fronds with fibrovascular cores, whereas these features are uncommon in high grade PIN (see below).

Pathologists may also disagree about whether the lesion is high grade PIN or high grade PIN and cancer. ${ }^{13}$ A good example of this situation is the presence of high grade PIN and only a few adjacent atypical glands. The issue is whether the small glands are outpouchings or tangential sections off the adjacent PIN gland, or whether they are microinvasive cancer. When the small atypical glands are too numerous and too crowded to be outpouchings or tangential sections off PIN glands, then infiltrating cancer can be diagnosed. Some 
pathologists think that the diagnosis of invasive cancer adjacent to high grade PIN can be based on the distance of the small atypical glands from the glands of PIN.$^{13}$ However, there is no agreement on the definition of this distance. In addition, immunohistochemical stains do not always aid in the solution of this problem. In fact, high grade PIN shows a discontinuous basal cell layer when labelled with the antibody to high molecular weight keratin, but even entirely benign glands may not always be labelled with this antibody. Other biomarkers, such as ploidy, do not discriminate between high grade PIN and prostatic carcinoma. ${ }^{17}$

Allam et al found that the variability in the identification of high grade PIN is related to the degree of interest in prostatic pathology, the conditions of the study, the subjective applications of diagnostic criteria, and the influence of peers and clinical colleagues. ${ }^{14}$ In particular, these authors discussed that variability is linked to the fact that the cut off point for high grade PIN can vary within a certain range in the continuous spectrum of PIN. For practising pathologists, translating descriptive terms such as frequent, numerous, few, and occasional into numerical values is difficult, and defining discrete compartments in a continuum of morphological changes is subjective and arbitrary. The variability in the diagnosis of high grade PIN that we see probably reflects these difficulties and uncertainties.

The management of the problems of translating descriptive terms into numerical values, and of uncertainties in the diagnosis and grading of PIN, have recently been considered by Montironi et al. ${ }^{18} 19$

\section{DIFFERENTIAL DIAGNOSIS}

The differential diagnosis of PIN includes several benign and malignant conditions. The former include atypia induced by inflammation, infarction, or radiation; transitional cell metaplasia; basal cell hyperplasia, with or without atypia; clear cell cribriform hyperplasia; and normal ejaculatory duct and seminal vesicle epithelium. ${ }^{1{ }^{20}}$ Malignant lesions that have to be distinguished from high grade PIN include transitional cell carcinoma involving prostate ducts and acini, and cribriform acinar and cribriform ductal prostatic carcinoma. Transitional cell carcinoma involving ducts and acini is usually a high grade tumour with pronounced cell pleomorphism, mitoses, and occasional comedonecrosis. Immunoreactivity for prostate specific antigen (PSA) and prostatic acid phosphatase (PAP) in proliferating cells is negative.

The cribriform pattern of Gleason grade 3 acinar prostatic carcinoma is histologically anomalous relative to the rest of the architectural scheme for grading invasive prostatic carcinoma. Like the other well and moderately differentiated (grade 1-3) carcinomas in the Gleason system, it is composed of individual glandular units that are separated by stroma. However, in all other grade 3 cancers, the neoplastic cells of each acinar unit typically have one pole abutting the stroma and an apical pole facing a single gland lumen. This pattern mim-

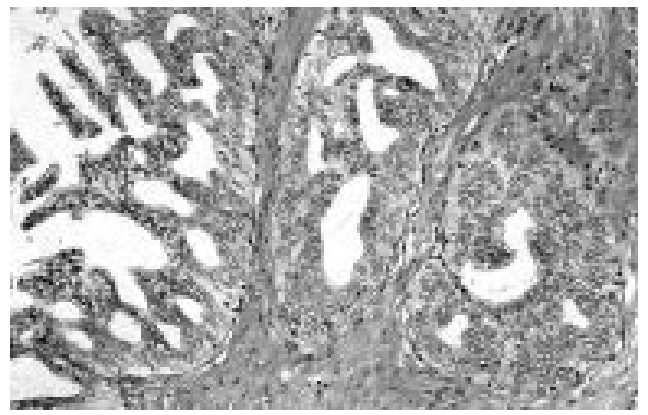

Figure 4 Inraductal carcinoma. The glandular unit is filled by a cell mass perforated by crisp, neatly punched out spaces of regular size.

ics normal glandular architecture. By contrast, in the cribriform grade 3 pattern, the potential lumen of each glandular unit is filled by a cell mass perforated by tiny lumens. This cribriform pattern of cancer cells is identical to that characterising one variant of Gleason grade 4 prostatic carcinoma, a pattern whose only distinguishing feature from grade 3 is that the cancer cells form large sheets with invasive borders.

Evidence has been presented that the histological features of the cribriform grade 3 pattern represent cancer growing within preexisting ducts and acini. Intraductal location is identified by recognition of the normal ductacinar branching pattern. This can be confirmed by the demonstration of a partially intact basal cell layer using high molecular weight keratin immunostaining (34 $\beta \mathrm{E} 12)$. The topographic analysis of $\mathrm{McNeal}$ et al indicates that the incipient lesion may spread rather rapidly and widely throughout the duct segment of origin, judging by the frequent involvement in continuity of lumens from near the urethra to the gland capsule. ${ }^{21}$ The same authors postulate that intraductal neoplasia affects the natural history of prostatic carcinoma by giving rise to (or being associated with) an unusual aggressive variant of invasive tumour, probably grade 4 or 5 in histological pattern.

This lesion was designated "intraductal carcinoma" by $\mathrm{McNeal}$ et $a .^{21}$ Other authors adopted the term of "high grade cribriform PIN". The latter term was based on the guidelines for the definition of high grade PIN, defining high grade PIN to include all dysplastic processes that still maintain a basal cell layer (fig 4). ${ }^{22-24}$

According to McNeal et al intraductal carcinoma usually arises within established invasive cancer, perhaps often from the same dysplastic focus that was previously the source of the original cancer. ${ }^{21} \mathrm{~A}$ consistent evolutionary sequence was traced in some of their cases. This included the initial step: gradual transition between normal epithelium and dysplasia. The next stage beyond dysplasia was focal epithelial accumulation into tufts, which developed into elongated pseudopapillations extending into the lumen. Then fusion of pseudopapillations into trabeculae or narrow septa composed of cuboidal cells in orderly arrangement is seen near the centre of the lumen, thus dividing the gland lumens into 
multiple elongate spaces (high grade dysplasia). Finally, increased cellularity of the trabeculae reduced the lumens to small round cribriform spaces (intraductal carcinoma). What is lacking in this last stage is the so called "maturation phenomenon", a feature thought to be characteristic of PIN lesions, namely: perimeter cells with features of clearly dysplastic cells, whereas, going from the periphery towards the centre, the nuclei become smaller and the nucleoli become less apparent. Mitoses and pleomorphism are infrequent in the cribriform pattern of high grade PIN and frequent in the cribriform pattern of acinar carcinoma (intraductal carcinoma). Comedo necrosis is extremely infrequent in high grade cribriform PIN and frequent in the cribriform pattern of acinar carcinoma.

Amin et al divided the cribriform glands seen in prostatic neoplasia into those with classic and those with non-classic architectural patterns based on Page's criteria for identifying ductal carcinoma in situ of the breast. ${ }^{25}$ Cribriform glands with crisp, neatly punched out spaces of regular size were considered classic glands, whereas slit like, irregular spaces were considered non-classic. Of the foci identified as PIN by Amin et $a l,{ }^{25} 20 \%$ of the cribriform glands had a classic pattern and $80 \%$ a non-classic pattern. By McNeal's criteria, these two patterns would be classified as intraductal carcinoma and high grade PIN, respectively. ${ }^{21} 26$

The incidence of intraductal carcinoma seen in radical prostatectomy specimens varies from study to study. Probably the best explanation for this variation concerns the criteria used to define cribriform PIN and whether immunohistochemistry was used to show the basal cell layer. McNeal and Yemoto ${ }^{26}$ found that the overall incidence of what they define as intraductal carcinoma was $30 \%$. Their study used immunohistochemistry only on a small proportion of cases. Rubin et al reported a frequency of $35 \%{ }^{24}$

In recent years other patterns of cytologically malignant intraductal proliferations have been identified: comedo, solid, and papillary. ${ }^{27} 28$ Wilcox et al found that these patterns are associated with a clinically aggressive prostatic carcinoma. ${ }^{28}$ These patterns, among which a cribriform one has to be included, are usually seen in association with prostatic ductal carcinoma (formerly endometrioid carcinoma) arising predominantly in large periurethral primary prostatic ducts, and with acinar prostatic carcinoma with focal ductal differentiation. Amin and colleagues ${ }^{25}$ attempted to identify distinctive criteria for the cribriform intraductal proliferation seen in ductal carcinoma, namely:

- The architectural pattern can be classic or non-classic.

- Mitoses are frequent.

- Pleomorphism is prominent.

- Comedo necrosis is very frequent.

A recent paper published by Weinstein ${ }^{29}$ gives further support to the concept of two populations of high grade PIN. This author examined the proliferative indices of benign epithelium, carcinoma, and non-cribriform high grade PIN found in close proximity to carcinoma. It was found that the proliferative index was higher for cancer than for high grade PIN, and that the latter fell into two distinct subgroups. It was concluded that there may be two types of lesions with the morphological appearance of high grade PIN, and that they may have a different biological relation to carcinoma. Weinstein ${ }^{29}$ put forward the hypothesis that some foci of high grade PIN are a consequence of carcinoma rather than being a precursor lesion, similar to intraductal carcinoma of the prostate.

There is no agreement in the literature about whether these patterns should be called high grade PIN, intraductal carcinoma, or intraductal spread of carcinoma. If we accept the guidelines for the definition of high grade PIN, then the term of PIN should be preferred so that a common terminology is used for such lesions. However, what is important is that the term we decide to used should be accompanied by the specific definition of the lesion or pattern being observed-for example, high grade cribriform PIN (intraductal carcinoma type). Even though we might not agree on the terminology, we usually agree on the fact that intraductal carcinoma, as well as the other intraductal patterns, might not be a preinvasive neoplastic condition, but instead a late event in tumour progression,,$^{30}$ as suggested by its strong association with poor prognostic factors, including tumour volume. Further supporting this view is the recent molecular evidence suggesting that intraductal carcinoma and Gleason 5 carcinoma have similar genetic alterations. ${ }^{32}$

\section{Possible subgroups of PIN}

HIGH GRADE PIN WITH UNUSUAL CELL TYPES

Three subgroups of high grade PIN with unusual cell types were described recently by Reyes et $a l^{33}$ namely: signet ring cell PIN, small cell PIN, and high grade PIN with mucinous secretion. All the cases were localised in the peripheral zone of the prostate. The first unusual type of PIN showed a tufted and micropapillary architectural growth pattern, with the constituent cells exhibiting a morphological appearance identical to that of the associated invasive signet ring cell carcinoma. The presence of basal cells was apparent by haematoxylin and eosin staining and was confirmed by immunohistochemistry. The intraductal and invasive signet ring cells were mucin negative and were immunoreactive for PSA.

A single case of small cell neuroendocrine high grade PIN was reported by the same authors. ${ }^{33}$ This case was characterised by mixed glandular (or secretory) and small cells and was identified in sections of a radical prostatectomy that harboured an invasive mixed adenocarcinoma-small cell carcinoma. In multiple foci a population of intraepithelial neoplastic cells morphologically identical to those in the invasive counterpart was present. The small cell PIN pattern was most often cribriform, with a striking polarisation of cellular differentiation, where the glandular cells were peripherally located and the small neoplastic 
cells were centrally located, with rosette like structures visualised. The intraductal small cell component in the centre of the cribriform glands had certain characteristics of neuroendocrine differentiation.

Reyes et al gave details of high grade PIN with mucinous features. ${ }^{33}$ This form of PIN was characterised by glands that were distended and filled with intraluminal blue mucin. This form was not associated with invasive mucinous carcinoma of the prostate but rather with the usual small acinar prostatic carcinoma. The most frequent architectural pattern of high grade PIN with mucinous features was the flat type. The intraluminal mucin was intensely PAS positive and Alcian blue positive in all cases.

The conclusion reached by Reyes et al was that the presence of these prostatic cell types in both high grade PIN and prostatic carcinoma provides support for a close relation between high grade PIN and the variants of invasive carcinoma of the prostate. ${ }^{33}$

HIGH GRADE PIN IN THE TRANSITION ZONE Epstein and colleagues ${ }^{34}$ and Quinn and colleagues $^{35}$ analysed the relation of high grade PIN to stages A and B prostatic carcinoma (in these two papers the term severe dysplasia was used). The former group of authors studied high grade PIN in totally embedded radical prostatectomy specimens and their TURPs from 32 stage A carcinomas. In $44 \%$ of the cases, high grade PIN was minimal in amount, $44 \%$ intermediate, and $12 \%$ extensive. High grade PIN was spatially associated with the main tumour in only $31 \%$ of cases. In $44 \%$ of the cases all of the high grade PIN was isolated (that is, it was not adjacent or intermingled with any carcinoma). In addition, high grade PIN in the TURP was seen in $16 \%$ of cases and was not predictive of the amount of tumour within the gland. This study confirms a general association between high grade PIN and the presence of carcinoma within the gland, and does not greatly support the concept that high grade PIN is a direct precursor of low grade stage A carcinomas located in the transition zone.

Quinn et al studied high grade PIN in totally embedded radical prostatectomy specimens from 40 stage $\mathrm{B}$, intermediate grade prostate carcinomas. ${ }^{35}$ All 40 cases had high grade PIN, which varied in amount. High grade PIN was predominantly concentrated in the peripheral region of the gland. Those cases with extensive high grade PIN had more multifocal small cancers compared with cases with intermediate or minimal severe dysplasia. Most dominant tumour nodules had some intermingled high grade PIN, and in $30 \%$ of the dominant tumour nodules, intermingled high grade PIN occupied $10-25 \%$ of the tumour nodule. High grade PIN was located next to the dominant tumour nodule in all cases. Nineteen percent of dominant nodules had extensive adjacent high grade PIN ranging from 5 to $14 \mathrm{~mm}$ in maximum diameter, showing that it should be possible to obtain only high grade PIN from needle biopsies of prostate cancers. According to the authors, these data show a close relation between high grade PIN and stage B prostatic carcinoma. In contrast, centrally located low grade multifocal cancers, as seen in stage A carcinoma, have a significantly weaker association with high grade PIN. ${ }^{34}$ Pacelli and Bostwick, ${ }^{36}$ Gaudin et $a l^{37}$ and Harvei and colleagues ${ }^{38}$ have confirmed this observation.

In addition, Montironi et al have investigated whether patients with high grade PIN of the transition zone have the same degree of association with prostatic carcinoma as patients with high-grade PIN of the peripheral/central zone. ${ }^{39}$ Their study was based on the expression of $\pi$-class glutathione $S$-transferase (GST- $\pi$ ) (see below). Two discrete immunostaining patterns were seen in high grade PIN. GST- $\pi$ expression similar to that of the normal tissue-stronger and more diffuse in the basal cell layer than in the luminal cell layer-was defined as pattern A. The stained basal cell layer was prominent at low magnification, partially and completely encircling acini and ducts containing PIN. The other pattern (pattern B) deviated from the normal and was characterised by an almost complete absence of GST- $\pi$ expression in the secretory cells and abundant expression in only scattered basal cells. Pattern A was seen more frequently in the transition than in the non-transition zone. Pattern B was mainly seen in high grade PIN of the non-transition zone in association with cancer. The differential expression of GST- $\pi$ in the transition and non-transition zones confirms the existence of two subgroups of high grade PIN, which have different spatial relations to carcinoma.

PUTATIVE PRENEOPLASTIC MARKERS WITH MINIMAL OR NO MORPHOLOGICAL CHANGES (OR PIN WITHOUT MORPHOLOGICAL CHANGES)

The link between atypical adenomatous hyperplasia (AAH) and cancer has not been demonstrated. Considering that high grade PIN of the transition zone is quite rare and its association with cancer of this zone is uncertain, the question one may ask is what are the characteristics of the precursor lesion(s) of the transition zone from which well differentiated prostatic carcinoma originates.

Recent studies have pointed out that the normal looking epithelium may show some molecular changes similar to those present in the preneoplastic and neoplastic lesions, both in the transition and non-transition zones. ${ }^{40}$ Here are two examples of such molecular changes, one concerning GST and the other telomerase activity.

The most studied function of the GST enzymes is their role in cellular detoxification. GSTs may prevent carcinogenesis through the inactivation of reactive electrophiles by conjugation to reduced glutathione. The most widely investigated with respect to human malignancy is GST- $\pi .^{41}{ }^{42}$ Immunohistochemical staining with anti-GST- $\pi$ antibodies is seen primarily in the cytoplasm of the basal cell layer of normal ducts and acini. Anti-GST- $\pi$ antibodies fail to detect the enzyme in most prostatic adenocarcinomas. It has been reported that the 
deoxycytidine residues in the 5'-regulatory CG island region of the GSTP1 gene, which encodes the human $\pi$ class GST, is commonly hypermethylated during prostatic carcinogenesis. ${ }^{43}{ }^{44}$ Deterioration is detectable in high grade PIN lesions and, to a minor degree, in some normal looking ducts and acini adjacent to either high grade PIN or to prostatic carcinoma. A similar pattern is also recognisable in normal tissue from prostates without preneoplastic and neoplastic lesions in occasional cases. ${ }^{40}$

Telomerase activity is considered to be a useful diagnostic marker of prostate cancer. ${ }^{45}$ Its expression is occasionally detected in benign prostatic tissue bordering prostate cancer and may result from either the presence of a primary undetected preneoplastic lesion or a secondary response of the benign elements to adjacent neoplastic lesions. The finding of telomerase activity in a certain proportion of biopsies from patients with benign prostatic hyperplasia is thought to be an early change in prostate carcinogenesis. It has been suggested that telomerase activity may be a useful biomarker for patients diagnosed with benign prostatic hyperplasia who may subsequently develop prostate cancer. ${ }^{46}$

These observations are related to the so called "enzyme altered foci" as putative preneoplastic markers. According to Pretlow and co-workers, the most abundant of these lesions with molecular alterations show minimal or no morphological changes. ${ }^{47}$

Changes occur also in the stroma surrounding ducts and acini with PIN. Montironi et al have shown that the degree of vascularisation (or angiogenesis) in normal prostate samples from total prostatectomies performed because of a preoperative diagnosis of prostatic carcinoma is close to that of low grade PIN. ${ }^{40} 48$

The transition from normal epithelium to prostate cancer without an intermediate morphological stage identifiable as PIN was considered possible in a recent paper on molecular genetics and the epidemiology of prostatic carcinoma (fig 5). ${ }^{49}$

\section{The morphological appearance of PIN after treatment}

The best way to eliminate the impact of cancer in humans is prevention, as long as this can be done with minimal risk or inconvenience. Although not without controversy, it follows that, if high grade PIN is indeed a precursor to invasive cancer, then the elimination, retardation, or reduction of high grade PIN would lead to a parallel reduction in cancer incidence.

The chemoprevention of premalignant prostatic lesions such as PIN is a strategy designed to inhibit or reverse the process of carcinogenesis by administering one or several noncytotoxic chemical compounds. A major pathway of chemoprevention unique to the prostate is the inhibition of androgen induced effects on prostate growth. In fact, overwhelming evidence suggests that androgens play an important role in the development and progression of prostate cancer. For more details on prostate cancer chemoprevention see Montironi et al. ${ }^{50}$

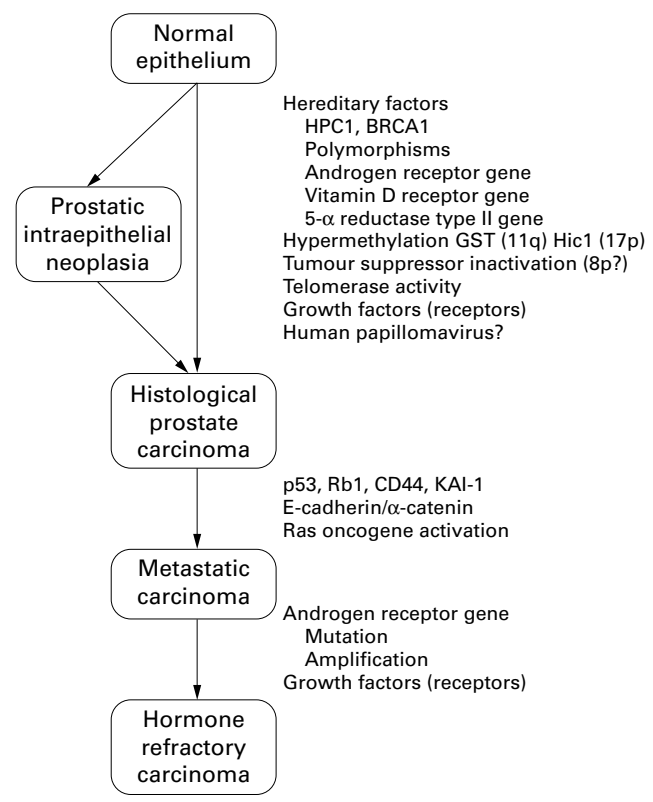

Figure 5 Transition from normal epithelium to prostate cancer with and without an intermediate morphological stage identifiable as prostatic intraepithelial neoplasia. ${ }^{49}$ GST, glutathione S-transferase; Rb1, retinoblastoma 1.

EFFECT OF ANDROGEN DEPRIVATION ON PIN Several papers have dealt with the effect of androgen manipulation on high grade PIN. ${ }^{51-54}$ A certain degree of secretory cell type stratification is always present. However, crowding is less evident than in untreated high grade PIN. The cells show cytoplasmic clearing and enlargement as a result of coalescence of vacuoles and rupture of cell membranes. The nuclei have different degrees of chromatin changes, ranging from a mild condensation-which barely allows the distinction between coarse chromatin granules (corresponding to heterochromatin) and finely dispersed chromatin (corresponding to euchromatin) - to a tightly condensed state close to that seen during apoptosis. ${ }^{51}$ Similar to treated prostatic carcinoma, apoptotic bodies are easily identifiable in all epithelial cell layers. ${ }^{52}$ The hallmark of all untreated high grade PIN is that the cells are frequently multinucleolated, the nucleoli being prominent, marginated, and with a perinucleolar halo. In treated cases, the nucleoli become inconspicuous, without margination, and have a smaller diameter. ${ }^{54}$ The duct and acinar lumen is always rich in cells: some are macrophages, some sloughed secretory cells with degenerative features, whereas others are apoptotic cells. The basal cell layer is easily recognisable in most instances (fig 6).

The severity of nuclear and cytoplasmic changes is not homogeneous among the PIN foci. Some foci in which the regressive changes are present only to a minor degree are still easily identifiable as having the features of high grade PIN. In addition to this, there seems to be some correspondence between the type of treatment and the degree of regressive changes. After total androgen ablation, morphological changes are more pronounced than after hormonal monotherapy; that is, luteinising hormone releasing hormone analogue or 


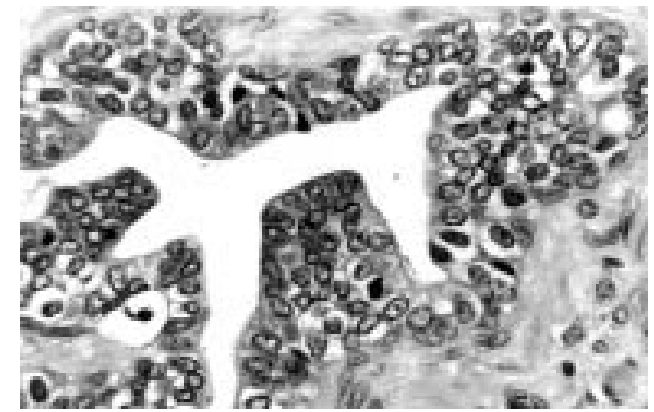

Figure 6 Prostatic intraepithelial neoplasia (PIN) following androgen ablation. Secretory cell type stratification is present. However, crowding is less evident than in untreated high grade PIN. The cells show cytoplasmic clearing and enlargement as a result of the coalescence of vacuoles and the rupture of cell membranes. The nucleoli are inconspicuous.

antiandrogen used alone. Chronic treatment with finasteride only has a small effect on the PIN cells. ${ }^{53}$

Treated PIN has to be distinguished from two benign alterations that affect the ducts and acini of the transition and central zones. One of these is basal cell hyperplasia. This is usually easy to distinguish from PIN because the cells composing the lesions have the typical morphological and immunohistochemical features of basal cells present in untreated prostates. In particular, there is a sharp transition between the basal cells and the secretory cells, the latter usually being in a single layer and with inconspicuous nucleoli, nuclear shrinkage, chromatin condensation, and cytoplasmic clearing. The other benign alteration that can mimic PIN is present in the central zone. Here, some ducts and acini show a certain degree of stratification of the benign looking secretory cells, which show regressive changes and bear some resemblance to the benign glands, with Roman bridge and cribriform patterns usually present in the central zone in untreated patients.

Treated PIN has to be distinguished from a lesion characterised by ducts and acini filled with neoplastic cells and with an almost continuous basal cell layer present at the periphery. The cells in this proliferation show very little regressive changes compared with those usually seen in the neoplastic cells infiltrating the surrounding stroma. In fact, the cells in the intraductal position still have a prominent nucleolus, whereas the cytoplasm has the features of the neoplastic cells either without or with little effect of the androgen ablation treatment. These dual effects on the cells in the two locations indicate that there is a differential response to treatment, this being more pronounced in the invasive counterpart. The primary Gleason grade present in the pretreatment biopsy is 3C. This ductal lesion seen after treatment should correspond to intraductal carcinoma (R Montironi et al, unpublished observations).

There is a pronounced decrease in the prevalence and extent of high grade PIN in prostates after androgen deprivation treatment compared with untreated prostates. Ferguson et al showed that the incidence of high grade
PIN is reduced from $83 \%$ in the prostatectomy only group to $50 \%$ among patients who received preoperative androgen deprivation treatment. ${ }^{55}$ In addition, PIN was present in more than two high power fields in only $21 \%$ of treated patients, compared with $67 \%$ of untreated patients. Similar results were reported by Vaillancourt et al..$^{54}$ These findings indicate that the dysplastic prostatic epithelium is hormone dependent. The two main effects of androgen ablation on the epithelial cells are to reduce proliferation and enhance apoptosis. ${ }^{56-59}$

\section{EFFECT OF RADIOTHERAPY ON PIN}

To date, there have been few papers on the effect of radiotherapy on high grade PIN. The most recent reference to this was made by Cheng et al in a paper on the prevalence and distribution of PIN in salvage radical prostatectomy specimens after radiotherapy. ${ }^{60}$ It was found that PIN identified after radiotherapy usually retains the features characteristic of untreated PIN. It is readily recognised in the radical prostatectomy specimens. The salient pathological features include nuclear crowding, nuclear overlapping and stratification, nuclear hyperchromasia, and prominent nucleoli. The basal cell layer is still present, but fragmented. The most common patterns of PIN are tufting and micropapillary, similar to those reported by Bostwick et al. ${ }^{7}$ Basal cell prominence and secretory cell cytoplasm vacuolisation may be seen in high grade PIN after radiotherapy.

The main differential diagnosis of high grade PIN after radiotherapy is atypical basal cell hyperplasia. The proliferation of basal cells in atypical basal cell hyperplasia is often eccentrically located with partial involvement of acini, retaining the overlying columnar or cuboidal secretory cells. These atypical basal cells have enlarged nuclei and nucleoli, fine powdery chromatin, occasional nuclear grooves, and an absence of apocrine blebs. A nuclear "bubble" artifact or intranuclear vacuoles may be seen. In difficult cases, the use of immunostaining for high molecular weight keratin might help to distinguish atypical basal cell hyperplasia from high grade PIN. Data on the effect of radiotherapy on intraductal carcinoma are not available yet.

Cheng et al also provided information about the prevalence and extent of PIN after radiotherapy. ${ }^{60}$ In particular, they found small volume PIN (mean, $0.12 \mathrm{~cm}^{3}$ ) in $62 \%$ of patients. By comparison, Qian et al noted that $82 \%$ of step sectioned prostate glands removed for prostate cancer had coexistent untreated high grade PIN, with an average volume of $1.32 \mathrm{~cm}^{3}{ }^{61}$ Akakura and colleagues ${ }^{51}$ found a higher incidence $(70 \%)$ of PIN after radiotherapy than did Cheng et al. ${ }^{60}$ These two groups suggested that radiotherapy affects non-invasive precursor lesions in a manner analogous to that seen in ductal carcinoma in situ of the breast. A different opinion was expressed by Wheeler, who concluded that there was insufficient information to determine whether radiotherapy affected the incidence and extent of PIN. ${ }^{62}$ 
Importance of high grade PIN

EVIDENCE LINKING HIGH GRADE PIN AND

PROSTATIC CARCINOMA

The following pieces of evidence link high grade PIN and prostatic carcinoma ${ }^{63}$ :

- The incidence and extent of PIN and prostatic carcinoma increase with patient age.

- There is an increased frequency, severity, and extent of PIN with prostatic carcinoma.

- PIN and prostatic carcinoma are both multifocal and share a similar location in the prostate zones.

- The transition of high grade PIN to prostatic carcinoma can be observed from the morphological point of view.

- High grade PIN has several features in common with prostatic carcinoma.

Several authors have reported an increasing frequency of PIN with advancing age and its association with prostatic carcinoma. Bostwick and Brawer ${ }^{6}$ showed that the frequency of PIN in prostates with cancer is significantly increased when compared with prostates not harbouring carcinoma. McNeal and Bostwick ${ }^{5}$ showed that PIN was present in $82 \%$ of step sectioned postmortem prostates with cancer, but only in $43 \%$ of benign prostates from patients of similar age. Qian et al found that $86 \%$ of a series of whole mount radical prostatectomy specimens with cancer contained high grade PIN, usually within $2 \mathrm{~mm}$ of the cancer. ${ }^{64}$ The severity of PIN in prostates with cancer was also increased when compared with those without. Both $\mathrm{McNeal}$ and Bostwick and Qian and colleagues ${ }^{64}$ have reported that PIN was more extensive in lower stage tumours, presumably because of an "overgrowth" or obliteration of PIN by larger high stage tumours.

The predominant location of PIN is the peripheral zone of the prostate where most moderately and poorly differentiated cancers arise. In fact, most foci of high grade PIN are exclusively in the peripheral zone (or nontransition zone; in one study, $63 \%$ ) or simultaneously in the peripheral and transition zones $(36 \%)$, and only rare cases $(1 \%)$ are exclusively in the transition zone. ${ }^{34}{ }^{35}$ Other authors have reported a higher frequency of encountering high grade PIN in the transition zone of the glands, with a range of $2-37 \%$ of the cases. $^{36-38}{ }^{63}$ Kovi and colleagues ${ }^{65}$ reported that the highest frequency of involvement of the transition zone $(37 \%)$ is in radical prostatectomies with cancer, although this finding was significantly lower in studies using TURPs.

With regard to multifocality, PIN is reported to be multifocal in $72 \%$ of radical prostatectomies with cancer, including $63 \%$ of those involving the non-transition zone and $7 \%$ of those involving the transition zone; $2 \%$ of the cases had concomitant single foci in all zones. $^{65-69}$

Little attention has been paid in the literature to the identification of the transition between high grade PIN and carcinoma. This topic was considered by $\mathrm{McNeal}$ et al in a paper dealing with microcarcinoma in the prostate and its association with duct-acinar dysplasia. ${ }^{66}$ Microcarcinomas were designated as cancers where the greatest dimension is $<4 \mathrm{~mm}$ on a single level of section, or those that occupy two adjacent levels with a sum of greatest dimensions $<5 \mathrm{~mm}$. Each microcarcinoma was classified according to the morphological features of its association with dysplasia (for example, PIN). In 48 of 107 tumours a small focus of newly formed, branching small glands with distinctive abnormal architecture and dysplastic epithelium formed an intermediate stage between the pre-existing ducts with dysplasia and frankly invasive carcinoma. These glands resembled a branching duct-acinar system in miniature and did not appear frankly invasive, despite their relatively small diameter and irregular branching pattern. They were lined by a crowded, pseudostratified epithelium similar to that seen in dysplasia, and contrasting with the simple columnar or cuboidal lining of the invasive grade 3 malignant glands. In all cases, the intermediate glands were located immediately adjacent to both dysplasia and invasive cancer and were at least partly interposed between the two.

In the study by McNeal et $a l^{66}{ }^{6}$ there were also some tumours showing direct invasion into the stroma by a small tubular malignant gland, which appeared to originate abruptly from a dysplastic duct wall (fig 7). This pattern is similar to that illustrated by others, ${ }^{570}$ and investigated recently by da Silva et al. ${ }^{71}$ They evaluated individual nuclei from high grade PIN lesions with early invasive carcinoma foci in the area of microinvasion and in the gland in which the microinvasion originated. Nuclei in the glandular epithelium were recorded sequentially, along the contour, at increasing distances from the point of microinvasion and by random selection in the region of microinvasion. The chromatin texture signatures showed a clear tend: there was an obvious attenuation as the measured nuclei approached the microinvasion area. At a distance $>60$ nuclear locations from the point of microinvasion, the nuclear signatures corresponded to those seen in high grade PIN. Between 40 and 20 nuclear locations removed from the microinvasion focus the signatures began to change gradually until at a distance of 15-5 locations they strongly resembled the signatures seen in adenocarcinoma. This observation was related to the fact that microinvasion might be linked to clonal selection and the emergence of clones that might be responsible for the invasive

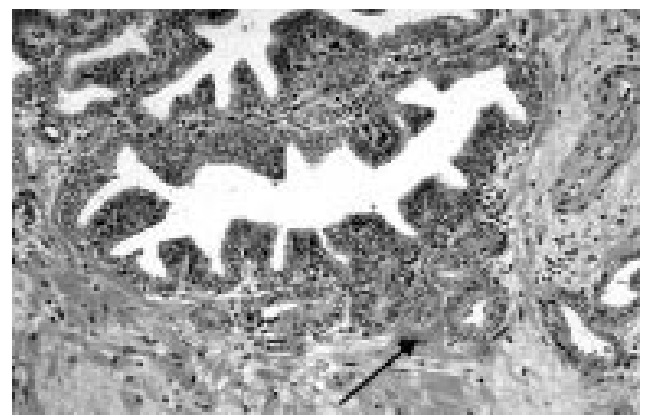

Figure 7 High grade prostatic intraepithelial neoplasia with early invasion. Direct invasion into the stroma by a small tubular malignant gland, which appeared to originate abruptly from a dysplastic duct wall (arrow). 
phenotype. A similar observation had been made for the transition from carcinoma in situ of the uterine cervix to invasive squamous carcinoma. $^{72}$ The observations made by McNeal and colleagues ${ }^{66}$ and by da Silva and colleagues $^{71}$ strongly support the concept of high grade PIN as a direct precursor of invasive adenocarcinoma.

Evidence supporting the relation of high grade PIN to prostatic carcinoma has also been found in immunohistochemical, morphometric, molecular, and genetic studies. ${ }^{567374}$ Virtually all studies of biomarkers have indicated that high grade PIN is related more closely to prostatic carcinoma than to benign epithelium. (For a comprehensive review on the biomarkers see Montironi et al. ${ }^{56}$ ) Evidence includes:

- Cell proliferation and death (apoptosis) are greater in PIN and prostatic carcinoma than in the normal prostate.

- PIN and prostatic carcinoma are phenotypically similar.

- PIN and prostatic carcinoma are morphometrically similar.

- There are common genetic alterations in PIN and prostatic carcinoma. The most common are: the gain of chromosome 7, particularly $7 \mathrm{q} 31$; loss of $8 \mathrm{p}$ and gain of $8 \mathrm{q}$; and loss of $10 \mathrm{q}, 16 \mathrm{q}$, and $18 \mathrm{q}$. Inactivation of tumour suppressor genes or overexpression of oncogenes in these regions might be important for the initiation and progression of prostate neoplasia. This strongly supports the hypothesis that PIN is the most likely precursor of prostatic carcinoma. ${ }^{32}$ 75-83

- The basal cell layer is disrupted in high grade PIN and is absent in prostatic carcinoma.

- Neovascularisation is greater in PIN and prostatic carcinoma than in the normal prostate.

PIN AS PREDICTOR OF MALIGNANCY IN NEEDLE BIOPSIES

High grade PIN is identified in $2-16.5 \%$ of needle biopsies. Its incidence probably varies according to the patient population under consideration (screening population $v$ urology office population). As an example, the American Cancer Society national cancer detection project identified PIN and cancer in $5.2 \%$ and $15.8 \%$ of men, respectively, from a series of 330 biopsies from men participating in an early detection project. ${ }^{84}$ Lee et al studied 256 ultrasound guided biopsies of hypoechoic lesions in a urology practice setting, and identified PIN and cancer in $10.5 \%$ and $40.2 \%$ of patients, respectively. ${ }^{85}$ Interestingly, those with PIN had a mean age of 65 years, whereas those with cancer had a mean age of 70 years. High grade PIN is encountered in up to $16.5 \%$ of contemporary needle biopsies in urology office practice. ${ }^{86}$

High grade PIN has a high predictive value as a marker of adenocarcinoma, and its identification in biopsy specimens warrants a search for concurrent invasive carcinoma. Davidson et al found adenocarcinoma in 35\% of subsequent biopsies from patients with a previous diagnosis of PIN, compared with $13 \%$ in a control group without PIN.$^{87}$ High grade PIN, patient age, and serum PSA concentration were all highly significant predictors of cancer, but PIN alone increased the risk 15-fold above those without PIN, and provides the highest risk ratio. Others have reported a high predictive value of PIN for cancer, ranging from 38\% to $100 \% \cdot{ }^{88-96}$ Approximately $50 \%$ of men with high grade PIN on biopsy will be found to have carcinoma on repeat biopsy within two years of follow up. These data underscore the strong association of high grade PIN and adenocarcinoma, and indicate that diagnostic follow up is needed. The cancer detection rate in patients with low grade PIN is identical to that in patients who underwent repeat biopsy for persistently raised serum PSA or because of an abnormal digital rectal examination..$^{93} 97$

Follow up is suggested at three or six month intervals for two years, and thereafter at 12 month intervals for life. Identification of PIN in the prostate should not influence or dictate therapeutic decisions other than chemoprevention.

\section{Summary remarks}

- High grade PIN is the most likely precursor of moderately and poorly differentiated prostatic carcinoma originating from the peripheral zone of the prostate.

- The histological definition of high grade PIN has gradually expanded to include almost every architecturally unremarkable gland with any growth pattern of neoplastic cells, so long as at least some basal cells can be identified. ${ }^{98}$ The different forms and patterns of PIN do not form discrete entities. In a certain proportion of cases these forms and patterns coexist and can also be contiguous, as if they were transitions from one to another. Malignant lesions that have to be distinguished from high grade PIN include transitional cell carcinoma involving prostate ducts and acini, and cribriform acinar and cribriform ductal prostatic carcinoma (intraductal carcinoma).

- High grade PIN has a high predictive value as a marker for carcinoma, and its identification in biopsy specimens warrants a search for concurrent invasive cancer.

- When low grade PIN is diagnosed on needle biopsy and repeat biopsies are performed, these patients are at no greater risk of having cancer than if their initial biopsy were reported as benign prostate tissue. Little information about low grade PIN as a precursor lesion is available in the current literature.

- The dysplastic prostatic epithelium composing PIN is hormone dependent. The two basic effects of androgen ablation on the epithelial cells consist of reducing proliferation and enhancing apoptosis.

$\mathrm{AAH}$ has been considered a premalignant lesion of the transition zone. However, a direct transition from AAH to cancer, as has been observed between PIN and cancer, has not been documented. We should look for another type of precursor lesion from which well differentiated prostatic carcinoma originates. 


\section{Notes added in proof}

(1) An additional subgroup of high grade PIN with unusual cell type was described in a recent publication (Berman DM, Yang J, Epstein JI. Foamy gland high-grade prostatic intraepithelial neoplasia. Am $\mathcal{F}$ Surg Pathol 2000;24:140-4).

(2) Excerpt from a publication based on the WHO Collaborative Project and Consensus Conference, Stockholm, June 8-9, 2000: "There are four other possible findings in the prostate (low-grade PIN, AAH, malignancy-associated foci, and atrophy) that may be premalignant, but the data for these are much less convincing than that for high grade PIN." (Bostwick D, Montironi R, Sesterhenn IA. Diagnosis of prostatic intraepithelial neoplasia. Scand $\mathcal{F}$ Urol Nephrol [In press.]).

1 Amin MB, Ro JY, Ayala AG. Putative precursor lesions of prostatic adenocarcinoma: fact or fiction? Mod Pathol 1993;6:476-83.

2 Orteil $\mathrm{H}$. Involutionary changes in prostate and female breast cancer in relation to cancer development. Can Med Assoc ₹ 1926;16:237.

3 Andrews GS. Latent carcinoma of the prostate. $\mathcal{F}$ Clin Pathol 1949;2:197.

4 McNeal JE. Morphogenesis of prostatic carcinoma. Cancer 1965;18:1659-66.

5 McNeal JE, Bostwick DG. Intraductal dysplasia: a premalignant lesion of the prostate. Hum Pathol 1986;17:64-71.

6 Bostwick DG, Brawer MK. Prostatic intra-epithelial neoplasia and early invasion in prostate cancer. Cancer 1987;59: 788-94.

7 Bostwick DG, Amin MB, Dundore P, et al. Architectural patterns of high grade prostatic intraepithelial neoplasia. patterns of high grade prostatic

8 Bostwick DG, Dousa M, Crawford B, et al. Neuroendocrine differentiation in prostatic intraepithelial neoplasia and adenocarcinoma. Am $\mathcal{F}$ Surg Pathol 1994;18:1240-6.

9 Di Sant'Agnese PA. Neuroendocrine differentiation in the precursors of prostate cancer. Eur Urol 1996;30:185-90.

10 Bonkhoff H, Stein U, Remberger K. Multidirectional differentiation in the normal, hyperplastic, and neoplastic human prostate: simultaneous demonstration of

11 Di Sant'Agnese PA. Neuroendocrine differentiation in prostatic adenocarcinoma does not represent true Paneth cell differentiation. Hum Pathol 1994;25:115-16.

12 Noordzij MA, Van Steenbrugge GJ, Van der Kwast TH, et al. Neuroendocrine cells in the normal, hyperplastic and al. Neuroendocrine cells in the normal, hyp
neoplastic prostate. Urol Res 1995;22:333-41.

13 Epstein JI, Grignon DJ, Humphrey PA, et al. Interobserver reproducibility in the diagnosis of prostatic intraepithelia neoplasia. Am f Surg Pathol 1995;19:873-86.

14 Allam CK, Bostwick DG, Hayes JA, et al. Interobserver variability in the diagnosis of high-grade prostatic intraepithelial neoplasia and adenocarcinoma. Mod Pathol 1996;9: $742-51$.

15 Tsuchiya K, Lynch J, True L. Prostatic intraepithelial neoplasia: intraobserver variability in grading. Mod Pathol 1993;6:71A.

16 Weinstein MH, Epstein JI. Significance of high grade prostatic intraepithelial neoplasia (PIN) on needle biopsy. Hum Pathol 1993;24:624-9.

17 Bostwick DG. High grade prostatic intraepithelial neoplasia. The most likely precursor of prostate cancer. Cance 1995;75:1823-36. 18 Montironi R, Mazzucchelli R, Bostwick DG, et al. Recent
advances in the quantitative morphological evaluation of prostate neoplasia. 7 Urol Pathol [In press.]

19 Montironi R, Mazzucchelli R, Santinelli A, et al. Case diagnosis as positive identification in prostatic neoplasia. Anal Quant Cytol Histol 1998;20:424-36.

20 Parkinson MC. Pre-neoplastic lesions of the prostate. Histopathology 1995;27:301-11.

21 McNeal EJ, Reese JH, Redwine EA, et al. Cribriform adenocarcinoma of the prostate. Cancer 1986;58:1714-19.

22 Algaba F, Epstein JI, Fabris G, et al. Working standards in prostatic intraepithelial neoplasia and atypical adenomatous hyperplasia. Pathol Res Pract 1995;191:836-7.

23 Algaba F, Trias I. Diagnostic limits in precursor lesions of prostate cancer. Eur Urol 1996;30:212-21.

24 Rubin MA, de La Taille A, Bagiella E, et al. Cribriform carcinoma of the prostate and cribriform prostatic intraepithelial neoplasia. Incidence and clinical implication. Am $\mathcal{F}$ Surg Pathol 1998;22:840-8.

25 Amin MB, Schultz DS, Zarbo RJ. Analysis of cribriform morphology in prostatic neoplasia using antibody to high-molecular-weight cytokeratins. Arch Pathol Lab Med 1994;118:260-4.
26 McNeal EJ, Yemoto CEM. Spread of adenocarcinoma within prostatic ducts and acini. Morphologic and clinical correlation. Am f Surg Pathol 1996;20:802-14.

27 Samaratunga $H$, Singh $M$. Distribution pattern of basal cells detected by cytokeratin 34 beta E12 in primary prostatic duct adenocarcinoma. Am f Surg Pathol 1997;21: 435-40.

28 Wilcox G, Soh S, Chakraborty S, et al. Patterns of high-grade prostatic intraepithelial neoplasia associated with clinically aggressive prostate cancer. Hum Patho 1998;29:1119-23.

29 Weinstein MH. Digital image analysis of proliferative index: two distinct population of high-grade prostatic intraepithelial neoplasia in close proximity to adenocarcinoma of the prostate. Hum Pathol 1998;29:620-6.

30 Bostwick DG, Kindrachuk R, Rouse R. Prostatic adenocarcinoma with endometrioid features. Clinical, pathologic, and ultrastructural findings. Am f Surg Pathol 1985;9:595609.

31 Bock BJ, Bostwick DG. Does prostatic ductal adenocarcinoma exist? Am f Surg Pathol 1999;23:781-5.

32 Qian J, Jenkins RB, Bostwick DG. Potential markers of aggressiveness in prostatic intraepithelial neoplasia detected by fluorescence in situ hybridization. Eur Urol 1996; 30:177-84.

33 Reyes AO, Swanson PE, Carbone JM, et al. Unusual histologic types of high-grade prostatic intraepithelial neoplasia. Am 7 Surg Pathol 1997;21:1215-22.

34 Epstein JI, Cho KR, Quinn BD. Relationship of severe dysplasia to stage A (incidental) adenocarcinoma of the prostate. Cancer 1990;65:2321-7.

35 Quinn BD, Cho KR, Epstein JI. Relationship of severe dysplasia to stage B adenocarcinoma of the prostate. Cancer 1990;65:2328-37.

36 Pacelli A, Bostwick DG. Clinical significance of high-grade prostatic intraepithelial neoplasia in transurethral resection specimens. Urology 1997;50:355-9.

37 Gaudin PB, Sesterhenn IA, Wojno KJ, et al. Incidence and clinical significance of high-grade prostatic intraepithelial neoplasia in TURP specimens. Urology 1997;49:558-63.

38 Harvei S, Skjorten FJ, Robsahm TE, et al. Is prostatic intraepithelial neoplasia in the transition/central zone a true precursor of cancer? A long-term retrospective study in Norway. Br f Cancer 1998;78:46-9.

39 Montironi R, Mazzucchelli R, Stramazzotti D, et al. Expression of $\pi$-class glutathione $S$-transferase: two populations of high-grade prostatic intraepithelial neoplasia with different relations to carcinoma. 7 Clin Pathol: Mol Pathol 2000;53: 122-8.

40 Montironi R, Hamilton PW, Scarpelli M, et al. Subtle morphological and molecular changes in normal-looking epithelium in prostates with prostatic intraepithelial neoplasia or cancer. Eur Urol 1999;35:468-73.

41 Baylin SB, Makos M, Wu JJ, et al. Abnormal patterns of DNA methylation in human neoplasia: potential consequences for tumor progression. Cancer Cells (Cold Spring Harbor) 1991;3:383-90.

42 Toffoli G, Viel A, Tumiotto L, et al. Expression of glutathione-S-transferase- $\pi$ in human tumours. Eur 7 Cancer 1992;28a: 1441-6.

43 Jarrard DF, Bova GS, Isaacs WB. DNA methylation, molecular genetic, and linkage studies in prostate cancer. Prostate 1996;6(suppl):36-44.

44 Lee WH, Morton RA, Epstein JI, et al. Cytidine methylation of regulatory sequences near the $\pi$-class glutathione S-transferase gene accompanies human prostatic carcinogenesis. Proc Natl Acad Sci U S A 1994;91:11733-7.

45 Kallabury BVS, Brien TP, Lowry CV, et al. Telomerase activity in human benign prostate tissue and prostatic adenocarcinoma. Diagn Mol Pathol 1997;6:192-8.

46 Scates DK, Muir GH, Venitt S, et al. Detection of telomerase activity in human prostate: a diagnostic marker for proase activity in human prostate: a diagnos.

47 Pretlow TG, Nagabhushan M, Pretlow TP. Prostatic intraepithelial neoplasia and other changes during promotion and progression. Pathol Res Pract 1995;191:842-9.

48 Montironi R, Magi-Galluzzi C, Diamanti L, et al. Prostatic intra-epithelial neoplasia. Qualitative and quantitative analyses of the blood capillary architecture thin tissue sections. Pathol Res Pract 1993;189:542-8.

49 Ruijter E, Van de Kaa C, Miller G, et al. Molecular genetics and epidemiology of prostate carcinoma. Endocr Rev 1999; 20:22-45.

50 Montironi R, Mazzucchelli R, Marshall JR, et al. Prostate cancer prevention: review of target populations, pathologicancer prevention: review of target populations, pathologi-
cal biomarkers, and chemopreventive agents. f Clin Pathol 1999;52:793-803.

51 Akakura K, Bruchovsky N, Goldemberg N, et al. Effects of intermittent androgen suppression on androgendependent tumors. Cancer 1993;71:2782-90.

52 Magi-Galluzzi C, Montironi R, Giannulis I, et al. Prostatic invasive adenocarcinoma. Effect of combination endocrine therapy (LHRH agonist and flutamide) on the expression and location of proliferating cell nuclear antigen (PCNA). Pathol Res Pract 1993;189:1154-60.

53 Montironi R, Diamanti L, Pomante R, et al. Prostatic intraepithelial neoplasia following six-month treatment with a 5 - $\alpha$-reductase inhibitor (finasteride). Anal Quant Cytol Histol 1996;18:461-71.

54 Vaillancourt L, Tetu B, Fradet Y, et al. Effect of neoadjuvant endocrine therapy (combined androgen blockade) on normal prostate and prostatic carcinoma. Am f Surg Pathol 1996;20:86-93. 
55 Ferguson J, Zincke H, Ellison E, et al. Decrease of prostatic intraepithelial neoplasia following androgen deprivation therapy in patients with stage T3 carcinom
radical prostatectomy. Urology $1994 ; 44: 91-5$.

56 Montironi R, Thompson D, Bartels PH. Premalignant lesions of the prostate. In: Lowe DG, Underwood JCE, eds. Recent advances in histopathology, 1999:147-72.

57 Armas OA, Aprikian AG, Melamed J, et al. Clinical and pathobiological effects of neoadjuvant total androgen ablation therapy on clinically localized prostatic adenocarcinoma. Am $\mathcal{F}$ Surg Pathol 1994;18:979-91.

58 Johnson MI, Robinson MC, Marsh C, et al. Expression of Bcl-2, p53 in high-grade prostatic intraepithelial neoplasia and localized prostate cancer: relationship with apoptosis and proliferation. Prostate 1998;37:223-9.

59 Montironi R, Schulman CC. Pathological changes in prostate lesions after androgen manipulation. $\mathcal{f}$ Clin Pathol 1998;51:5-12.

60 Cheng L, Cheville JC, Pisansky TM, et al. Prevalence and distribution of prostatic intraepithelial neoplasia in salvage radical prostatectomy specimens after radiation therapy. Am ₹ Surg Pathol 1999;23:803-8.

61 Qian J, Wollan P, Bostwick DG. The extent and multicentricity of high-grade prostatic intraepithelial neoplasia in clinically localized prostatic adenocarcinoma. Hum Pathol 1997;28:143-8

62 Wheeler TM. Influence of irradiation and androgen ablation on prostatic intraepithelial neoplasia. Eur Uro 1996;30:261-4.

63 Sakr WA, Grignon DJ, Haas GP, et al. Age and racial distribution of prostatic intraepithelial neoplasia. Eur Urol 1996; 30:138-44.

64 Qian J, Bostwick DG, Takahashi S, et al. Chromosomal anomalies in prostatic intraepithelial neoplasia and carcinoma detected by fluorescence in situ hybridization. Cancer Res 1995;55:5408-14.

65 Kovi J, Jackson MA, Heshmat MY. Ductal spread in prostatic carcinoma. Cancer 1985;56:1566-73.

$66 \mathrm{McNeal}$ JE, Villers A, Redwine EA, et al. Microcarcinoma in the prostate: its association with duct-acinar dysplasia. Hum Pathol 1991;22:644-52.

67 Qian J, Bostwick DG. The extent and zonal location of prostatic intraepithelial neoplasia and atypical adenomatous hyperplasia: relationship with carcinoma in radica prostatectomy specimens. Pathol Res Pract 1995;191: $860-7$

68 Kovi J, Mostofi FK, Heshmat MY, et al. Large acinar atypical hyperplasia and carcinoma of the prostate. Cancer 1988;61:555-61.

69 Algaba F, Trias I, Lopez L, et al. Neuroendocrine cells in peripheral prostatic zone: age, prostatic intraepithelial neoplasia and latent cancer-related changes. Eur Urol 1995;27: 329-33.

70 McNeal JE. The significance of duct-acinar dysplasia in prostatic carcinogenesis. Prostate 1988;13:91-102.

71 da Silva VD, Montironi R, Thompson D, et al. Chromatin texture in high grade prostatic intraepithelial neoplasia and early invasive carcinoma. Anal Quant Cytol Histol 1999;21: early invasi $113-20$.

72 Boehm N, Sandritter W. DNA in human tumors: a cytophotometric study. Curr Top Pathol 1975;60:151-219.

$73 \mathrm{McNeal}$ JE, Haillot O, Yemoto C. Cell proliferation in dysplasia of the prostate: analysis by PCNA immunostaining. Prostate 1995;27:258-68.

74 Qian J, Jenkins RB, Bostwick DG. Detection of chromosomal anomalies and c-myc gene amplification in the cribriform pattern of prostatic intraepithelial neoplasia and carcinoma by fluorescence in situ hybridization. Mod Pathol 1997;10:1113-19.

75 Alers JC, Krijtenberg PJ, Vissers KJ, et al. Interphase cytogenetics of prostatic adenocarcinoma and precursor lesions: analysis of 25 radical prostatectomies in 17 adjacent prostatic intraepithelial neoplasias. Genes Chromosomes Cancer 1995;12:241-50.

76 Bergerheim USR, Kunimi K, Collins VP, et al. Deletion mapping of chromosomes 8,10 and 16 in human prostatic mapping of chromosomes 8,10 and 16 in human prostatic
77 Bova GS, Carter BS, Bussemarkers MJG, et al. Homozygous deletion and frequent allelic loss of chromosome 3869-73.

78 Cunningham JM, Shan A, Wick MJ, et al. Allelic imbalance and microsatellite instability in prostatic adenocarcinoma. Cancer Res 1996;56:4475-82.

79 Emmert-Buck MR, Vocke CD, Pozzati RO, et al. Allelic loss on chromosome $8 \mathrm{p} 12-21$ in microdissected prostatic intraepithelial neoplasia (PIN). Cancer Res 1995;55:2959-

80 MacGrogan D, Levy A, Bostwick DG, et al. Loss of chromosome $8 \mathrm{p}$ loci in prostate cancer: mapping by quantitative allelic balance. Genes Chromosomes Cancer 1994;10: $151-9$.

81 Qian J, Bostwick DG, Takahashi S, et al. Chromosomal anomalies in prostatic intraepithelial neoplasia and carcinoma detected by fluorescence in situ hybridization. Cancer Res 1995;55:5408-14.

82 Sakr WA, Macoska JA, Benson P, et al. Allelic loss in locally metastatic, multisampled prostate cancer. Cancer Res 1994; 54:3273-7.

83 Takahashi S, Qian J, Brown JA, et al. Potential markers of prostate cancer aggressiveness detected by fluorescence in situ hybridization in needle biopsies. Cancer Res 1994;54: 3574-9.

84 Mettlin C, Lee F, Drago J, et al. The American Cancer Society national prostate cancer detection project. Findings on the detection of early prostate cancer in 2425 men. Cancer 1991;67:2949-58.

85 Lee F, Torp-Pedersen ST, Carroll JT, et al. Use of transrectal ultrasound and prostate-specific antigen in diagnosis of prostatic intraepithelial neoplasia. Urology 1989;24(suppl): $4-12$.

86 Haddad FS. Risk factors for perineal seeding of prostate cancer after needle biopsy. F Urol 1990;143:587-8.

87 Davidson D, Bostwick DG, Qian J, et al. Prostatic intraepithelial neoplasia is a risk factor for adenocarcinoma: thelial neoplasia is a risk factor for adenocarcinoma: predictive

88 Aboseif S, Shinohara K, Weidner N, et al. The significance of prostatic intra-epithelial neoplasia. Br F Urol 1995;76: $355-9$.

89 Bostwick DG, Iczkowski KA. Minimal criteria for the diagnosis of prostate cancer on needle biopsy. Ann Diagn Pathol 1997;1:104-29.

90 Markham CW. Prostatic intraepithelial neoplasia. Detection and correlation with invasive cancer in fine-needle biopsy. Urology 1989;24(suppl):57-61.

91 Brawer MK, Bigler SA, Sohlberg OE, et al. Significance of prostatic intraepithelial neoplasia on prostate needle biopsy. Urology 1991;38:103-7.

92 Berner A, Danielsen HE, Pettersen EO, et al. DNA distribution in the prostate. Normal gland, benign and premalignant lesions, and subsequent adenocarcinomas. Anal Quant Cytol Histol 1993;15:247-52.

93 Keetch DW, Humphrey P, Stahl D, et al. Morphometric analysis and clinical follow-up of isolated prostatic intraepithelial neoplasia in needle biopsy of the prostate. $\mathcal{F}$ Urol 1995;154:347-51.

94 Langer JE, Rovner ES, Coleman BG, et al. Strategy for repeat biopsy of patients with prostatic intraepithelial neoplasia detected by prostate needle biopsy. F Urol 1996;155:228-31.

95 Raviv G, Zlotta AR, Janssen TH, et al. Does prostate specific antigen and prostate-specific antigen density enhance the detection of prostate cancer in patients initially diagnosed to have prostatic intraepithelial neoplasia? Cancer 1996;77: 2103-8.

96 Shepherd D, Keetch DW, Humphrey PA, et al. Repeat biopsy strategy in men with isolated prostatic intraepithelial neoplasia on prostate needle biopsy. F Urol 1996;156:460-3.

97 Raviv G, Janssen TH, Zlotta AR, et al. Prostatic intraepithelial neoplasia: influence of clinical and pathological data on the detection of prostate cancer. F Urol 1996;156:1-6.

98 Pedersen J, Maruniak NA, Murphy WM. Can basal cell exist in carcinomatous prostatic glands? F Urol Pathol 1999; $10: 1-8$ 